\title{
CLINICAL COMPARISON OF ANAEROBIC BLOOD-CULTURE MEDIA FOR DETECTING BACTERAEMIA DUE TO VIRIDANS STREPTOCOCCI AND ORAL ANAEROBES
}

\author{
D. C. Shanson, I. Moule* and M. Tadayon \\ Department of Medical Microbiology, Westminster Medical School and ${ }^{*}$ Department of \\ Oral Surgery, Westminster Hospital, London SWIP $2 A R$
}

\begin{abstract}
Summary. Brain heart infusion cysteine broth, with and without Panmede ${ }^{\circledR}$ (a papain digest of ox liver) and Fastidious Anaerobe Broth, with and without Liquoid, were compared by inoculating the broths with blood collected from each of 51 patients, 2 min after dental extraction. Bacteraemia caused by viridans streptococci or oral nonsporing anaerobes or both was detected in 39 patients $(76 \%)$. Detection of bacteraemia caused by viridans streptococci and anaerobes was more rapidly achieved by the addition of Panmede to brain heart infusion broth. Significantly more cases of bacteraemia caused by viridans streptococci were detected by use of the Panmede-containing medium than by use of Fastidious Anaerobe Broth after incubation of the broths for only 1 day. Use of brain heart infusion cysteine broth with and without Panmede, and Fastidious Anaerobe Broth permitted detection of bacteraemia caused by viridans streptococci in 26,11 and 22 patients respectively during incubation for 2 weeks. Bacteraemia caused by anaerobes was detected by use of these three media in 24, 13 and 23 patients respectively. The addition of Liquoid to Fastidious Anaerobe Broth had no significant effect on the detection of bacteraemia caused by viridans streptococci or anaerobes.

The Panmede-containing blood-culture medium should be a useful anaerobic broth in the investigation of patients with suspected endocarditis, because viridans streptococci are also rapidly detected.
\end{abstract}

\section{INTRODUCTION}

Anaerobic blood culture media vary greatly in their ability to support the growth of small inocula of non-sporing anaerobes (Forgan-Smith and Darrell, 1974; Shanson, 1974; Shanson and Barnicoat, 1975; Szawatkowski, 1976; Collee, Duerden and Brown, 1977; Barr, 1980). Conflicting results have been obtained, especially when media containing thioglycollate have been tested, but good recovery of anaerobes in simulated blood cultures have consistently been obtained with Fastidious Anaerobe Broth, which also contains thioglycollate (Ganguli, Turton and Tillotson, 1982; Hunt 
and Price, 1982; Shanson and Pratt, 1983). Good recovery of Bacteroides fragilis was achieved when cysteine $0.05 \%$ was added to brain heart infusion broth (Shanson and Singh, 1981). A blood-culture medium containing a papain digest of ox liver, Panmede ${ }^{\circledR}$, was suggested as particularly suitable for paediatric blood cultures (Hunt and Price, 1982) and a subsequent controlled study demonstrated a statistically significant improvement in the recovery of non-sporing anaerobes when Panmede $2.5 \%$ was added to brain heart infusion broth containing cysteine $0.05 \%$ (Shanson and Pratt, 1983). All these reports have been based on in-vitro experiments.

Reliable recovery of viridans streptococci from blood cultures is important for the microbiological diagnosis of infective endocarditis but there has been no published comparison of these media for detecting bacteraemia caused by viridans streptococci. We decided to compare the abilities of three recently introduced media to detect viridans streptococci and oral non-sporing anaerobes by inoculating blood collected from patients undergoing dental extraction. The media tested were brain heart infusion broth containing cysteine, with and without Panmede, and Fastidious Anaerobe Broth.

Because sodium polyanethol sulphonate (Liquoid) may inhibit anaerobic streptococci in certain blood culture media (Shanson, 1974), a further objective of the present study was to evaluate the effect of adding Liquoid $0.05 \%$ to Fastidious Anaerobe Broth on the detection of post-extraction bacteraemia.

\section{MATERIALS AND METHODS}

Blood culture media. Brain heart infusion broth (Bacto 0037-01, Difco) was prepared by adding $37 \mathrm{~g}$ of powder to $500 \mathrm{ml}$ of distilled water. L-Cysteine (BDH Chemicals Ltd, Poole, Dorset) $0.5 \mathrm{~g}$ was dissolved in $5 \mathrm{ml}$ of $\mathrm{N} \mathrm{HCl}$ and the $5 \mathrm{ml}$ of solution was added to $500 \mathrm{ml}$ of broth. The $p \mathrm{H}$ was adjusted to 7.4 and the final volume made up to $1 \mathrm{~L}$. The broth was dispensed in $75-\mathrm{ml}$ volumes in bottles with perforated metal caps and rubber liners. The medium was autoclaved at $121^{\circ} \mathrm{C}$ for $15 \mathrm{~min}$.

To prepare the medium containing Panmede (a papain digest of ox liver; Paines and Byrne, Greenford, Middlesex), $50 \mathrm{~g}$ of the substance was added to $50 \mathrm{ml}$ of distilled water and mixed; $4.6 \mathrm{ml}$ of $10 \mathrm{~N} \mathrm{NaOH}$ was added and the solution was made up to $100 \mathrm{ml}$ with distilled water. To $1 \mathrm{~L}$ of brain heart infusion broth containing cysteine $0.05 \%$ was added $50 \mathrm{ml}$ of the $50 \%$ Panmede solution. The $p \mathrm{H}$ was adjusted to 7.8 with $10 \mathrm{~N} \mathrm{HCl}$ and the broth was dispensed and autoclaved as before. The $p \mathrm{H}$ of the medium was checked after autoclaving and found to be 7.4.

Media prepared in the laboratory were used within 2 weeks of preparation.

Fastidious Anaerobe Broth with thymidine (Lab M Ltd, Salford, Lancashire) was supplied commercially in bottles containing $75 \mathrm{ml}$ of broth. A similar broth containing Liquoid $0.05 \%$ was also obtained commercially from Lab M Ltd.

Patients and blood-culture-collection methods. Adults undergoing dental extraction were included in the trial if there was no history of a susceptible heart lesion and if no antibiotics had been given in the previous week. Most of the extractions were carried out under general anaesthesia. Approximately $2 \mathrm{~min}$ after completion of extraction of the first tooth, $20 \mathrm{ml}$ of venous blood was collected aseptically from the ante-cubital fossa. Each of the four blood-culture media were inoculated with $5 \mathrm{ml}$ of blood via the rubber diaphragm in the top of each bottle. The media were always inoculated in the same order for the first 25 patients and the order was reversed for the subsequent 26 patients.

Processing of blood cultures. The blood-culture broths were inspected daily and the bottles were subcultured after incubation for 1, 2, 5, 7, 10,14 and 21 days. At each subculture two fresh $5 \%$ blood-agar plates were inoculated with $0.03 \mathrm{ml}$ of mixed culture fluid with a calibrated Pasteur pipette. One blood-agar plate was also inoculated with Staphylococcus aureus to help detect any so-called 'satellite' streptococci; this plate was incubated aerobically in a $\mathrm{CO}_{2}$ 
incubator for up to 3 days. The other blood-agar plate was incubated for 3 days, in an anaerobic atmosphere containing $\mathrm{CO}_{2} 10 \%$ in an anaerobic jar (Don Whitley, Shipley, West Yorkshire); a $2 \cdot 5-\mu \mathrm{g}$ metronidazole disk was applied at the primary inoculum site to assist in the rapid detection of anaerobes.

Identification of isolates. Suspected anaerobes were subcultured aerobically and anaerobically for up to 5 days in an atmosphere containing $\mathrm{CO}_{2} 10 \%$; isolates were also inoculated into Robertson's cooked meat broth for further identification tests. Isolates which grew only anaerobically and were sensitive to metronidazole were confirmed as anaerobes. The colonial morphology and reaction with Gram's stain of each anaerobe were noted and antibiotic sensitivity tests were performed with an anaerobe identification Mastring (Diamed Diagnostics Ltd, Bootle, Merseyside) that included penicillin 2-unit, erythromycin $60-\mu \mathrm{g}$, rifampicin 15- $\mu \mathrm{g}$, colistin $10-\mu \mathrm{g}$, kanamycin $1000-\mu \mathrm{g}$ and vancomycin $5-\mu \mathrm{g}$ disks. The antibiotic-susceptibility pattern of each anaerobe was used for presumptive identification (Leigh and Simmons, 1977).

Alpha- or non-haemolytic gram-positive cocci that grew aerobically, were resistant to optochin, were catalase- and coagulase-negative, and failed to hydrolyse aesculin in a medium containing $40 \%$ bile, were identified as viridans streptococci unless Lancefield grouping by the Streptex test (Oxoid) suggested that the isolate belonged to Lancefield Group D. Strains of viridans streptococci were further identified by the API 20 streptococcal identification test (API Laboratory Products Ltd, Basingstoke, Hampshire).

\section{RESULTS}

Bacteraemia was detected in $39(76 \%)$ of 51 patients $c .2$ min after dental extraction. Viridans streptococci alone were isolated in seven cases and anaerobes alone in seven cases; aerobes mixed with anaerobes were detected in 25 patients. The addition of Panmede $2.5 \%$ to brain heart infusion cysteine broth significantly increased the number of cases of dental bacteraemia detected: 21 cases were detected by use of brain heart infusion cysteine broth without Panmede compared with 33 cases detected by use of broth containing Panmede $(\mathrm{p}<0.05)$ (table I). There was no difference in the total number of cases of dental bacteraemia detected by brain heart infusion cysteine broth supplemented with Panmede (33 cases) and Fastidious Anaerobe Broth ( 33 cases). The addition of Liquoid $0.05 \%$ to $75 \mathrm{ml}$ of Fastidious Anaerobe Broth had no effect on the number of cases of bacteraemia detected within 2 weeks (table I).

TABLE 1

Comparison of blood culture media for the detection of viridans streptococci and anaerobes

\begin{tabular}{l|rrrrr}
\hline & \multicolumn{5}{c}{$\begin{array}{c}\text { Number (and percentage) of cases of } \\
\text { bacteraemia detected in }\end{array}$} \\
\cline { 2 - 6 } \multicolumn{1}{c}{ Organisms } & BHICP & BHIC & FAB & FAB +L & All 4 media \\
\hline Viridans streptococci & $26(51)$ & $11(22)$ & $22(43)$ & $24(47)$ & $32(63)$ \\
$\begin{array}{l}\text { Non-sporing anaerobes } \\
\text { Mixture of anaerobes } \\
\text { and streptococci }\end{array}$ & $24(47)$ & $13(25)$ & $23(45)$ & $28(55)$ & $32(63)$ \\
All organisms & $17(33)$ & $3(6)$ & $12(23)$ & $19(37)$ & $25(49)$ \\
\hline
\end{tabular}

$\mathrm{BHICP}=$ brain heart infusion cysteine broth supplemented with Panmede $2.5 \%$; $\mathrm{BHIC}=$ brain heart infusion cysteine broth; $\mathrm{FAB}=$ Fastidious Anaerobe Broth; FAB $+\mathrm{L}=$ Fastidious Anaerobe Broth with Liquoid $0.05 \%$. 


\section{Detection of viridans streptococci}

The medium supplemented with Panmede detected bacteraemia caused by viridans streptococci more quickly than the same medium without Panmede or the Fastidious Anaerobe Broth (with or without Liquoid) (table II). The greatest difference was observed after incubation of the media for only 1 day, at which time streptococci were isolated from Panmede-containing broth in ten cases and from Fastidious Anaerobe Broth in four cases. This difference was statistically significant $(p<0.05)$. Liquoid did not significantly improve the detection of streptococcal bacteraemia when added to Fastidious Anaerobe Broth.

Table III shows the identity of streptococci isolated from each broth within 7 days of inoculation. Streptococcus mitis was isolated more frequently from the Panmedecontaining medium and from the Fastidious Anaerobe Broth which included Liquoid. The difference between medium with and without Panmede was statistically significant $(\mathrm{p}<0.05)$.

When the broths were incubated for 10-21 days, six isolates of viridans streptococci failed to grow on repeated subculture from each of the two new media and three

TABLE II

Comparison of rapidity of detection of viridans streptococci

\begin{tabular}{c|crrrr}
\hline & Number of cases of viridans bacteraemia \\
Duration of incubation $\begin{array}{c}\text { dect in } \\
\text { (days) }\end{array}$ & \multicolumn{5}{|c}{} \\
\cline { 2 - 6 } & BHICP & BHIC & FAB & FAB + L & All 4 Media \\
\hline 1 & 10 & 2 & 4 & 6 & 12 \\
2 & 20 & 10 & 18 & 15 & 26 \\
4 & 25 & 11 & 22 & 22 & 31 \\
7 & 26 & 11 & 22 & 24 & 32 \\
\hline
\end{tabular}

For abbreviations see table I.

TABLE III

Identity of viridans Streptococci isolated from each medium during incubation for 7 days

\begin{tabular}{l|rrrr}
\hline \multirow{2}{*}{\multicolumn{1}{c|}{ Organism }} & \multicolumn{4}{|c}{ Number of isolates from } \\
\cline { 2 - 5 } & BHICP & BHIC & FAB & FAB + L \\
\hline Streptococcus mitis & 12 & 4 & 7 & 11 \\
Streptococcus sanguis II & 11 & 3 & 11 & 11 \\
'Satellite' streptococci & 2 & 3 & 2 & 1 \\
Streptococcus milleri & 1 & 2 & 2 & 1 \\
Streptococcus salivarius & 1 & 0 & 1 & 0 \\
Unidentified streptococcus & 1 & 0 & 0 & 1 \\
Total & 28 & 12 & 23 & 25 \\
\hline
\end{tabular}

For abbreviations see table I. 
isolates lost viability in brain heart infusion cysteine broth without Panmede. One isolate of a 'satellite' streptococcus lost viability in the latter medium after incubation of the broth for only 3 days.

\section{Detection of anaerobic bacteraemia}

Anaerobes were detected more frequently in the Panmede- and Liquoid-containing media than in the other two broths after incubation for 2 days (table IV). However, the difference was not statistically significant. There was no difference in the numbers of cases of anaerobic bacteraemia detected by use of the Panmede-containing medium or Fastidious Anaerobe Broth during incubation for 2-10 days. However the addition of Panmede $2.5 \%$ to brain heart infusion cysteine broth significantly increased the number of cases detected throughout the 14-day incubation period $(\mathrm{p}<0.05)$. The addition of Liquoid to Fastidious Anaerobe Broth was not associated with a significant increase in the number of cases detected during incubation for 2-7 days. There was a suggestion that the addition of Liquoid increased the recovery of gram-negative anaerobes, particularly Veillonella spp., Bacteroides ureolyticus and $B$. oralis. Two strains of anaerobic streptococci were isolated only from broth without Liquoid (table V).

There was no loss of viability of anaerobes during the first week of incubation, but after incubation for 2 weeks, 13 anaerobes failed to grow on subculture of the Panmede-containing broth and nine failed to grow on subculture of Fastidious Anaerobe Broth.

\section{Detection of polymicrobial bacteraemia.}

There was no significant difference in the ability of the four media to detect polymicrobial bacteraemia caused by anaerobes. Polymicrobial bacteraemia, caused by viridans streptococci mixed with at least one anaerobe, was detected in 17 cases by use of the Panmede-containing broth; in three cases by use of brain heart infusion cysteine broth; in 12 cases by use of Fastidious Anaerobe Broth; and in 19 cases by use of Fastidious Anaerobe Broth with Liquoid.

TABLE IV

Comparison of rapidity of detection of anaerobes

\begin{tabular}{c|rrrrc}
\hline & \multicolumn{5}{|c}{ Number of cases of anaerobic bacteraemia detected } \\
in \\
$\begin{array}{c}\text { Duration of incubation } \\
\text { (days) }\end{array}$ & BHICP & BHIC & FAB & FAB + L & All 4 media \\
\cline { 2 - 6 } & 8 & 2 & 4 & 9 & 14 \\
\hline 2 & 16 & 9 & 16 & 21 & 26 \\
7 & 22 & 12 & 22 & 25 & 31 \\
10 & 24 & 13 & 23 & 28 & 32 \\
14 & & & & & \\
\hline
\end{tabular}

For abbreviations see table I. 
TABLE V

Identity of anaerobic bacteria isolated from each medium during incubation for 2 weeks

\begin{tabular}{l|rrrr}
\hline & \multicolumn{4}{|c}{ Number of isolates from } \\
\cline { 2 - 5 } \multicolumn{1}{c}{ Organism } & BHICP & BHIC & FAB & FAB + L \\
\hline Bacteroides oralis & 13 & 4 & 10 & 11 \\
Bacteroides melaninogenicus & 4 & 5 & 6 & 6 \\
Bacteroides ureolyticus & 2 & 0 & 1 & 3 \\
Bacteroides sp. & 0 & 0 & 1 & 1 \\
Total Bacteroides isolates & 19 & 9 & 18 & 21 \\
Veillonella spp. & 7 & 6 & 8 & 11 \\
Anaerobic streptococci & 3 & 1 & 4 & 2 \\
Propionibacterium spp. & 0 & 2 & 0 & 1 \\
Total isolates & 29 & 18 & 30 & 35 \\
\hline
\end{tabular}

For abbreviations see table I.

\section{DISCUSSION}

The present study has demonstrated for the first time a clear clinical advantage for adding Panmede $2.5 \%$ to an anaerobic blood culture medium, brain heart infusion cysteine broth, for the detection of bacteraemia caused by both viridans streptococci and non-sporing anaerobes. This confirms previous results with simulated blood cultures (Shanson and Pratt, 1983). It is not known which of the many growth factors included in the liver digest, Panmede, is responsible for the effects observed. Although the clinical performance of brain heart infusion cysteine broth containing Panmede was superior to that of Fastidious Anaerobe Broth for the rapid detection of viridans streptococci, there was no significant difference between the two media for the detection of anaerobes. However, the Panmede-containing medium is less expensive than the Fastidious Anaerobe Broth used in this study.

The addition of Liquoid $0.05 \%$ to Fastidious Anaerobe Broth made no difference to the detection of viridans streptococci although Liquoid is recommended for inclusion in blood-culture broths for the isolation of aerobes (Von Haebler and Miles, 1938; Stokes, 1974; Washington, 1982). Because each 5-ml blood sample was diluted $c$. 1 in 17 in broth it is likely that the antibacterial effect of fresh human blood against viridans streptococci was minimal (Roome and Tozer, 1968) so that the role of Liquoid in reducing further the antibacterial effect of blood was limited. However the results suggest that Liquoid may slightly improve the detection rate of bacteraemia caused by gram-negative dental anaerobes, such as Veillonella spp. and B. ureolyticus, despite the dilution factor. Simulated blood culture studies with media containing thioglycollate and $B$. fragilis had previously suggested that a 1 in 17 dilution of human blood in broth without Liquoid was sufficient (Shanson, 1974). It is possible that Veillonella spp. and certain oral Bacteroides spp. are more susceptible to fresh blood than B. fragilis. Bacteraemia caused by anaerobic streptococci was detected in only four patients by use of Fastidious Anaerobe Broth without Liquoid and in two of these cases the parallel 
broths with Liquoid failed to isolate these organisms. This suggests that gram-positive anaerobic cocci may sometimes be inhibited by Liquoid, which supports previous in-vitro observations (Shanson, 1974). However, the difference observed in the present study between the rates of recovery of anaerobes from broths with and without Liquoid was not statistically significant.

We are grateful to Janet Pratt for technical assistance during the early part of this study.

\section{REFERENCES}

Barr J G 1980 A cooked meat blood culture medium; shelf life and clinical evaluation compared with other systems. Journal of Infection 2:247-258.

Collee J G, Duerden B I, Brown R 1977 Recovery of anaerobic bacteria from small inocula: a model for blood culture studies. Journal of Clinical Pathology 30:609-614.

Forgan-Smith W R, Darrell J H 1974 A comparison of media used in vitro to isolate non-sporing Gram-negative anaerobes from blood. Journal of Clinical Pathology 27:280-283.

Ganguli L, Turton L J, Tillotson G S 1982 Evaluation of fastidious anaerobe broth as a blood culture medium. Journal of Clinical Pathology 35:458-461.

Hunt G H, Price E H 1982 Comparison of a homemade blood culture broth containing a papain digest of liver, with four commercially available media for the isolation of anaerobes from simulated paediatric blood cultures. Journal of Clinical Pathology 35:1142-1149.

Leigh D A, Simmons K 1977 Identification of non-sporing anaerobic bacteria. Journal of Clinical Pathology 30:991-992.

Roome A P C H, Tozer R A 1968 Effect of dilution on the growth of bacteria from blood cultures. Journal of Clinical Pathology 21:719-721.

Shanson D C 1974 An experimental assessment of different anaerobic blood culture methods. Journal of Clinical Pathology 27:273-279.

Shanson D C, Barnicoat M 1975 An experimental comparison of thiol broth with Brewer's thioglycollate for anaerobic blood cultures. Journal of Clinical Pathology 28:407-409.

Shanson D C, Pratt J 1983 Effect of adding a papain digest of ox liver to brain heart infusion cysteine broth on the recovery of non-sporing anaerobes from simulated blood cultures. Journal of Clinical Pathology 36:678-682.

Shanson D C, Singh J 1981 Effect of adding cysteine to brain-heart infusion broth on the isolation of Bacteroides fragilis from experimental blood cultures. Journal of Clinical Pathology 34:221-223.

Stokes E J 1974 Blood culture technique. Association of Clinical Pathologists Broadsheet no. 81.

Szawatkowski M V 1976 A comparison of three readily available types of anaerobic blood culture media. Medical Laboratory Sciences 33:5-12.

Von Haebler T, Miles A A 1938 The action of sodium pol'y anethol sulphonate ('Liquoid') in blood cultures. Journal of Pathology and Bacteriology 46:245-252.

Washington J A 1982 The role of the microbiology laboratory in the diagnosis and antimicrobial treatment of infective endocarditis. Mayo Clinic Proceedings 57:22-32. 